\title{
Evaluación longitudinal mediante resonancia magnética del modelo de esquizofrenia de lesión neonatal en el hipocampo ventral
}

\author{
Longitudinal magnetic resonance evaluation of the schizophrenia model of neonatal \\ lesion in the ventral hippocampus
}

\begin{abstract}
Alma D. Genis-Mendoza1,2, Carina Elizalde-Martínez', José J. Martínez-Magaña², Brenda Cabrera-Mendoza', Axayacatl Morales-Guadarrama³, Emilio Sacristán ${ }^{3}$, Isabel Beltrán-Villalobos ${ }^{4}$, Carlos A. Tovilla-Zarate ${ }^{4,5}$ y Humberto Nicolini ${ }^{\text {* }}$

${ }^{1}$ Instituto Nacional de Medicina Genómica, Secretaría de Salud, Ciudad de México; ${ }^{2}$ Servicio de Atención Psiquiátrica, Hospital Psiquiátrico Infantil Juan N. Navarro, Secretaría de Salud, Ciudad de México; ${ }^{3}$ Centro Nacional de Imagenología e Instrumentación Médica (Cl3M), Universidad Autónoma Metropolitana, Iztapalapa, Ciudad de México; ${ }^{4}$ Universidad Autónoma de la Ciudad de México, Ciudad de México; ${ }^{5}$ Unidad Multidisciplinaria de Comalcalco, Universidad Juárez Autónoma de Tabasco, Comalcalco. México
\end{abstract}

\section{Resumen}

Objetivo: Evaluar mediante resonancia magnética (RM) la progresión de la lesión en el modelo de esquizofrenia de lesión del núcleo del hipocampo ventral (LNHV). Método: Se realizaron RM en ratas Wistar macho, desde los 8 días posnatales hasta los 139 días, en animales con LNHV y sin lesión (sham). Las RM se realizaron con un equipo Variant de 7 T. Los datos se analizaron con el software Amira para un análisis de morfometría basada en vóxels. Resultados: Observamos hiperseñales con un realce significativo en las estructuras analizadas en el grupo con LNHV, y mayor volumen en los ventrículos laterales, presentando un mayor tamaño de la lesión el día PD96 y significativamente reducido en el día PD139. Conclusiones: Encontramos un reacomodo celular durante la progresión de la lesión, lo cual podría ser efecto de la activación de las células inmunitarias.

Palabras clave: Esquizofrenia. Lesión neonatal del hipocampo ventral. Resonancia magnética.

\begin{abstract}
Objective: To evaluate the progression by means of nuclear magnetic resonance of the lesion in the schizophrenia model of lesion of the ventral hippocampal nucleus ( $L V N H)$. Method: Magnetic resonance imaging (MRI) were performed in male Wistar rats, from 8 days postnatal to 139 days, in animals with LNHV and without lesion (sham). The MRI were carried out on a Variant 7 T equipment. The data were analyzed with the Amira software, for a voxel-based morphometric analysis. Results: We observed the presence of hypersignals with a significant enhancement in the structures analyzed in the group with LVNH, and greater volume in the lateral ventricles, presenting a larger size of the lesion on day PD96 and significantly reducing on day PD139. Conclusions: We found a cell rearrangement during the progression of the lesion, which could be the effect of the activation of immune cells.
\end{abstract}

Key words: Schizophrenia. Neonatal ventral hippocampal lesion. Magnetic resonance imaging.

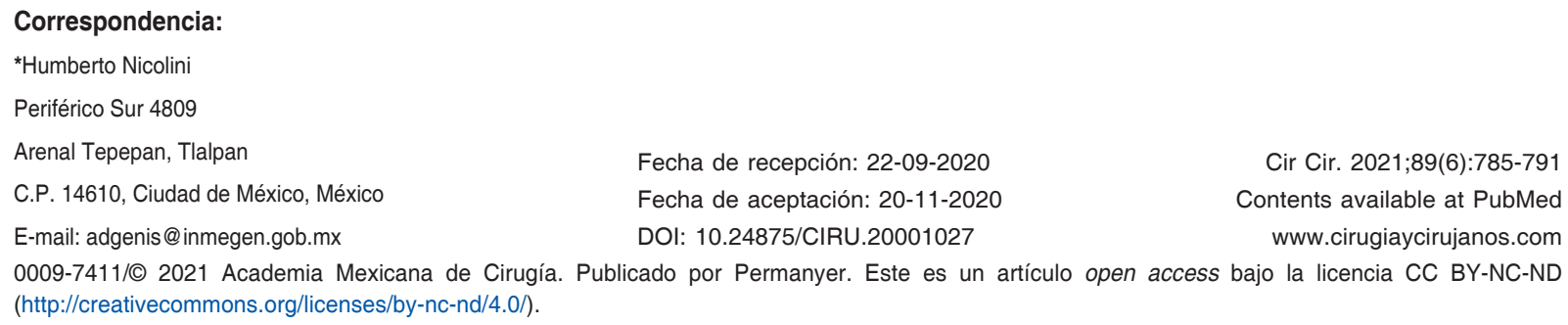




\section{Introducción}

La esquizofrenia es un trastorno neuropsiquiátrico que afecta a más de 21 millones de personas en el mundo'. En México, 1,100,000 personas padecen esquizofrenia, ocupando el quinto lugar en la lista de padecimientos de mayor discapacidad ${ }^{2}$. A pesar de la relevancia de este trastorno, hasta el momento no se ha elucidado su etiología. Sin embargo, se han propuesto varias hipótesis, como la del neurodesarrollo, la cual postula que al menos una parte de las personas con esquizofrenia presentan alteraciones estructurales en el sistema nervioso central producidas en etapas tempranas del desarrollo ${ }^{3}$. Algunas anomalías estructurales se han identificado en estudios post mortem, mientras que otras se han identificado en individuos vivos diagnosticados con el trastorno, principalmente por tecnologías de neuroimagen, como la tomografía computarizada y la resonancia magnética (RM). Un hallazgo constante en los estudios de RM en los pacientes con esquizofrenia es el alargamiento de los ventrículos laterales ${ }^{3}$. Los estudios de RM estructural sugieren que las anomalías cerebrales de los pacientes están presentes desde el inicio de la enfermedad. Sin embargo, los estudios longitudinales son de utilidad para evaluar la progresión y los cambios morfológicos, pero se han visto limitados por muestras pequeñas e intervalos de seguimiento cortos o poco frecuentes ${ }^{4}$.

Sin embargo, para poder probar tratamientos y explorar aspectos neurobiológicos que podrían ser de interés en la esquizofrenia se debe contar con modelos que permitan traducir la sintomatología. Dentro de los modelos de esquizofrenia se encuentran, entre otros, el modelo de lesión neonatal del hipocampo ventral (LNHV) y el modelo de mutación de Disc $1^{5}$. El modelo de LNVH es ampliamente utilizado, ya que ha proporcionado reproducciones confiables de las anormalidades químicas y de comportamiento observadas en los pacientes con esquizofrenia, incluyendo síntomas positivos y negativos ${ }^{6}$. Uno de los aspectos relevantes de este modelo es la reproducción de anomalías cerebrales anatómicas observadas en los humanos ${ }^{7}$. Por lo anterior, el modelo de LNVH ofrece la oportunidad de evaluar la progresión de los cambios morfológicos cerebrales asociados a la esquizofrenia.

El objetivo del presente trabajo fue evaluar los cambios morfológicos longitudinales en los cerebros de ratas Wistar macho con lesión en el hipocampo ventral, incluyendo tamaño y existencia de cavitación, especialmente en el hipocampo y ventrículos desde el día 1 posterior a la lesión (PD) hasta el día PD139 desde el nacimiento, mediante siete mediciones por RM.

\section{Método}

\section{Modelo animal}

Se incluyeron 30 ratas Wistar macho que fueron divididas en dos grupos: uno con LNHV y el otro como control. Al primer grupo de 15 ratas se les realizó una administración intracraneal de $0.3 \mu \mathrm{l}$ de ácido iboténico a la edad de 7 días (grupo LNVH), mientras que a las 15 ratas del grupo control se les realizó una administración intracraneal de $0.3 \mu \mathrm{l}$ de solución salina de buffer de fosfatos. Ambos procedimientos se realizaron con el procedimiento descrito por Genis-Mendoza, et al. ${ }^{8}$ La lesión neonatal se realizó en el hipocampo ventral: las crías de 5-7 días de vida fueron anestesiadas por hipotermia colocándolas en hielo durante 12-15 min; posteriormente, se colocaron en un estereotáxico con adaptador especial para animales neonatos. A cada cría se le realizó una incisión en la piel del cráneo y se le administraron $0.3 \mu \mathrm{l}$ de ácido iboténico (disuelto en buffer de fosfatos $0.1 \mathrm{M}, \mathrm{pH}$ 7.4) directamente en el hipocampo ventral durante 2 minutos con una cánula de acero inoxidable de $30 \mathrm{Ga}$ de grosor. Las coordenadas fueron: anteroposterior $2.0 \mathrm{~mm}$, mediolateral $\pm 2.5 \mathrm{~mm}$ relativo al bregma y ventrodorsal $-3.3 \mathrm{~mm}$ de la duramadre. Posteriormente los animales se suturaron y se dejaron recuperar bajo el calor indirecto de una lámpara de $40 \mathrm{~W}$ hasta recuperar su temperatura. Los animales operados se regresaron con sus madres hasta el momento del destete a los 21 días de edad.

El cuidado de los animales se realizó de acuerdo con la Norma Oficial Mexicana NOM-062-ZOO-1999 y la Guía Internacional para el cuidado de los animales NRC 2002. Los animales se mantuvieron bajo las siguientes condiciones: ciclos de 12/12 horas de luz/ oscuridad, humedad relativa de $45-60 \%$, 12 a 15 ciclos de ventilación y una temperatura de $18-26^{\circ} \mathrm{C}$.

El presente proyecto fue revisado y aprobado por el Comité de Ética en Investigación del Instituto Nacional de Medicina Genómica, con número de registro 120/2014/I.

\section{Monitoreo de la lesión in vivo por resonancia magnética}

La evaluación por RM se realizó en las siguientes edades: PD8, PD27, PD48, PD69, PD96 PD120 y PD139. A partir del PD27, los animales fueron anestesiados con 
Tabla 1. Comparación de los volúmenes $\left(\mu \mathrm{m}^{3}\right)$ obtenidos mediante segmentación en las siete sesiones de resonancia magnética

\begin{tabular}{|c|c|c|c|c|c|c|c|c|}
\hline & Grupo & PD8 & PD27 & PD48 & PD69 & PD96 & PD120 & PD139 \\
\hline \multirow[t]{2}{*}{ Cerebro } & LNHV & 1375.07 & 1325.18 & 1718.15 & 1719.01 & 1724.41 & 1736.28 & 1744.98 \\
\hline & Control & 1461.86 & 1473.66 & 1626.59 & 1645.57 & 1696.35 & 1657.51 & 1688.03 \\
\hline \multirow[t]{2}{*}{ Cuarto ventrículo } & LNHV & 2.19 & 2.53 & 3.56 & 6.79 & 5.8 & 4.81 & 6.06 \\
\hline & Control & 1.45 & 1.48 & 2.64 & 3.56 & 5.87 & 6.06 & 6.92 \\
\hline \multirow[t]{2}{*}{ Tercer ventrículo } & LNHV & 2.41 & 1.54 & 9.43 & 10.02 & 11.67 & 10.81 & 6.92 \\
\hline & Control & 1.91 & 2.14 & 2.31 & 2.83 & 3.16 & 5.67 & 5.73 \\
\hline \multirow[t]{2}{*}{ Hipocampo derecho } & LNHV & 33.68 & 28.8 & 31.84 & 38.43 & 39.68 & 37.24 & 38.89 \\
\hline & Control & 15.93 & 25.73 & 29.99 & 31.42 & 37.05 & 37.18 & 42.19 \\
\hline \multirow[t]{2}{*}{ Hipocampo izquierdo } & LNHV & 29.45 & 26.98 & 32.04 & 39.81 & 39.29 & 36.91 & 40.08 \\
\hline & Control & 17.19 & 25.24 & 25.38 & 30.98 & 37.9 & 40.21 & 43.24 \\
\hline \multirow[t]{2}{*}{ Ventrículo lateral derecho } & LNHV & 12.77 & 2.58 & 7.71 & 3.03 & 3.36 & 6.92 & 9.03 \\
\hline & Control & 0.55 & 0.88 & 1.71 & 2.24 & 2.97 & 3.56 & 3.69 \\
\hline \multirow[t]{2}{*}{ Ventrículo lateral izquierdo } & LNHV & 9.6 & 2.63 & 6.86 & 3.49 & 7.58 & 6.79 & 10.42 \\
\hline & Control & 0.66 & 1.04 & 2.5 & 2.86 & 3.16 & 3.23 & 3.76 \\
\hline \multirow[t]{2}{*}{ Lesión derecha } & LNHV & 0 & 0.77 & 0.46 & 3.1 & 3.56 & 1.91 & 0.99 \\
\hline & Control & 0 & 0 & 0 & 0 & 0 & 0 & 0 \\
\hline \multirow[t]{2}{*}{ Lesión izquierda } & LNHV & 0.66 & 1.38 & 0.73 & 3.1 & 4.48 & 1.78 & 0.99 \\
\hline & Control & 0 & 0 & 0 & 0 & 0 & 0 & 0 \\
\hline
\end{tabular}

LNHV: lesión neonatal en el hipocampo ventral.

Los datos a partir de PD8 son medias de solo dos animales que continuaron el estudio, mientras que en el tiempo PD8 son medias de todos los animales incluidos.

una mezcla de $1.7 \mathrm{mg} / \mathrm{kg}$ de peso de ketamina y $1.8 \mathrm{mg} / \mathrm{kg}$ de peso de xilacina, mientras que los animales a la edad de PD8 fueron anestesiados con hielo. Los roedores se colocaron en un cilindro inmovilizador para ingresar al equipo de RM. Las imágenes se obtuvieron con un equipo Varian $7 \mathrm{~T}$ en el Centro Nacional de Investigación en Imagenología e Instrumentación Médica de la Universidad Autónoma Metropolitana. Las imágenes ponderadas en T2 se obtuvieron utilizando una adquisición FSEMS con los siguientes parámetros: tiempo de eco $65 \mathrm{~ms}$, tiempo de repetición 8,200 ms, seis promedios por ciclo y ángulo de giro de $180^{\circ}$. El campo de visión fue de $22.5 \times 20 \mathrm{~mm}^{2}$, en una matriz de $256 \times 256$ que resultó en una resolución de $88 \times 78$ umpp. Se realizaron 45 cortes contiguos $(400 \mu \mathrm{m}$ de grosor) y el tiempo de exploración aproximado fue de 25 minutos por animal.

\section{Análisis de los datos de las imágenes de resonancia magnética}

El análisis de las imágenes de RM se centró en diferentes regiones de interés, incluyendo el hipocampo, los ventrículos y la lesión en el hipocampo ventral. Debido a las medidas ponderadas en T2 utilizadas en el presente estudio, los ventrículos laterales y el tejido lesionado se observaron como hiperintensos debido al líquido cefalorraquídeo y a la presencia de agua libre en estas áreas. Se analizaron cada hemisferio e hipocampo por animal realizando la segmentación de las imágenes con el software Amira $5.3 .3^{9}$, con el fin de obtener las medidas de la lesión (del grupo LNHV y del grupo control), el hipocampo, el tercer ventrículo y los ventrículos laterales mediante el uso de morfometría basada en vóxeles.

\section{Resultados}

Con la finalidad de hacer la comparación de los diferentes volúmenes del cerebro, se obtuvieron las siguientes mediciones: cuarto ventrículo (4to $\mathrm{V}$ ), tercer ventrículo (3er V), hipocampo $(H)$, ventrículos laterales (VL derecho e izquierdo) y tamaño de la lesión promovida por el ácido iboténico (Tabla 1). Las partes segmentadas que se presentan en la figura 1 son el tercer y el cuarto ventrículos, el hipocampo, la lesión 


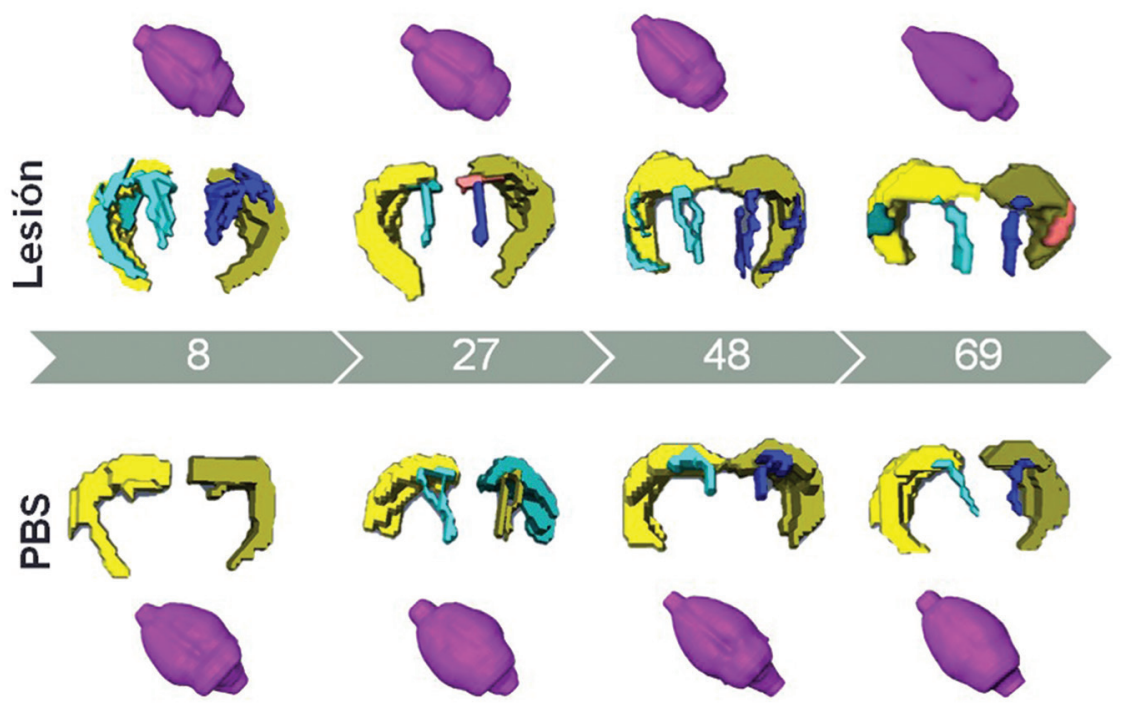

Figura 1. Imágenes segmentadas de resonancia magnética del cerebro del grupo de animales con lesión neonatal en el hipocampo ventral y del grupo control (PBS) en diferentes evaluaciones según la edad (PD8, PD27, PD48 y PD69). Se distinguen en color morado el cerebro completo, en amarillo el hipocampo derecho e izquierdo, en verde la lesión derecha y en rosa la lesión izquierda, en turquesa el ventrículo izquierdo, y en azul rey, el ventrículo derecho.

y los ventrículos laterales para las edades PD8, PD27, PD48 y PD69, y en la figura 2 se presentan las edades PD96, PD120 y PD139.

El volumen de los ventrículos laterales fue cualitativamente mayor en los animales del grupo de LNHV que en los del grupo control en las siete diferentes edades evaluadas, en ambos hemisferios. Con respecto al hipocampo, el volumen también fue cualitativamente mayor en el grupo de LNHV que en el grupo control para todas las regiones, aunque en las primeras mediciones, aparentemente, no había cambios morfológicos detectables (Tabla 1).

Con respecto a la lesión, en las primeras mediciones era imperceptible. Sin embargo, a partir de la segunda medición en el PD27, la lesión se comienza a vislumbrar, específicamente en el ventrículo izquierdo. Para el PD48 la lesión fue más marcada, con diferencias en tamaño (por ejemplo, $0.73 \mu \mathrm{m}^{3}$ para la lesión izquierda y $0.46 \mu \mathrm{m}^{3}$ para la lesión derecha) (Fig. 1), llegando a su máximo tamaño en el día PD96 (con $4.48 \mu \mathrm{m}^{3}$ en la lesión izquierda y $3.56 \mu \mathrm{m}^{3}$ para la lesión derecha), reestructurándose el tejido de la lesión en las dos siguientes mediciones (Tabla 1).

Adicionalmente, uno de los hallazgos más interesantes fue la presencia de hiperseñales con un realce significativo en las estructuras analizadas en el grupo con LNHV. La ausencia de dichas hiperseñales en el grupo control sugiere que es poco probable que se deban a la acumulación del líquido inyectado. Las hiperseñales observadas pudieran reflejar gliosis y edema $^{10}$, dos procesos que a menudo se producen simultáneamente, o en un patrón en serie, posterior a un daño neuronal.

\section{Discusión}

En el presente trabajo se hizo una comparación cualitativa longitudinal de la lesión en el modelo de esquizofrenia de LNHV. Se encontraron hiperseñales en el grupo con LNHV, pero no en el grupo control, las cuales podrían corresponder a áreas en las que se presenta una reacción glial grave, posiblemente debido a la rotura de la barrera hematoencefálica ${ }^{10}$ (Figs. 1-2). El aumento glial también podría estar relacionado con el proceso de neuroinflamación en individuos con esquizofrenia ${ }^{11,12}$, caracterizado por una cascada compleja que involucra la activación microglial por señalización de citocinas ${ }^{12}$. Se ha caracterizado un incremento de la neuroinflamación en numerosos estudios de individuos con esquizofrenia, y se ha propuesto como un factor posiblemente involucrado en la etiología de la enfermedad ${ }^{13,14}$. Otro hallazgo que podría estar relacionado con un proceso neuroinflamatorio es el mayor volumen ventricular en el grupo de LNHV, comparado con el grupo control, lo cual podría ser efecto de la exposición neonatal a citocinas proinflamatorias. Se ha observado una correlación positiva significativa entre la exposición fetal 

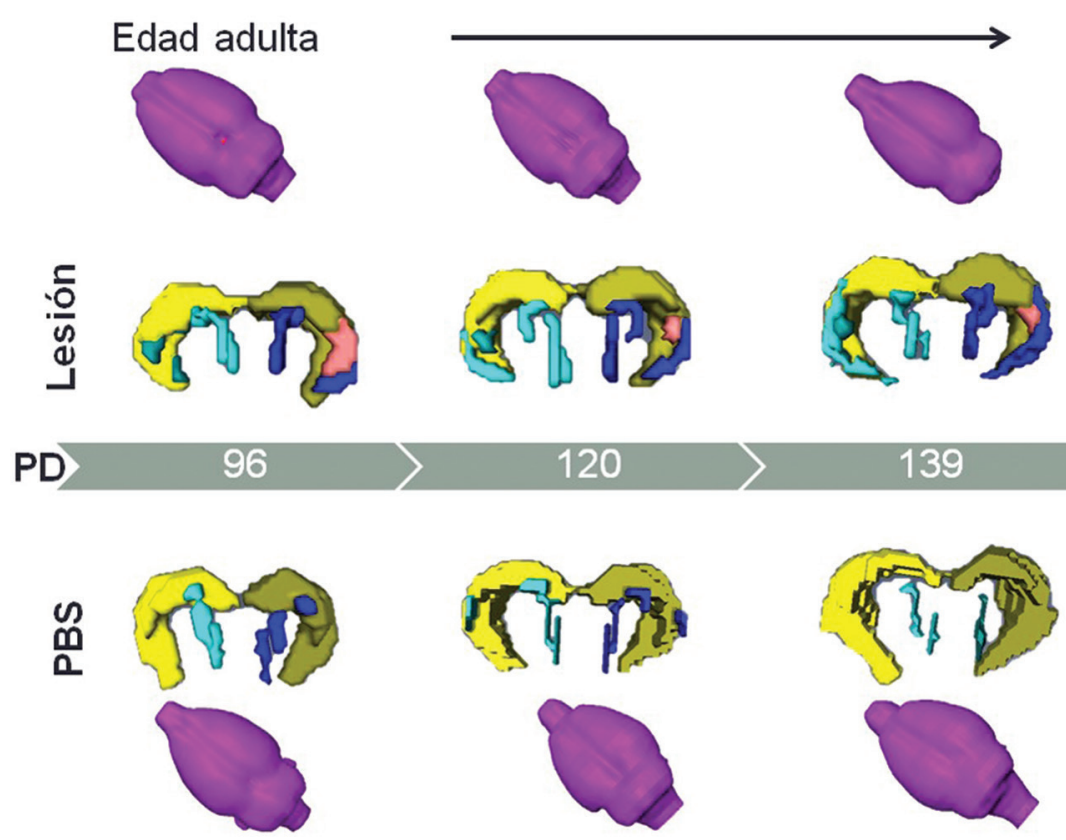

Figura 2. Imágenes segmentadas de resonancia magnética del cerebro del grupo de animales con lesión neonatal en el hipocampo ventral y del grupo control (PBS) en diferentes evaluaciones según la edad (PD96, PD120 y PD139). Se distinguen en color morado el cerebro completo, en amarillo el hipocampo derecho e izquierdo, en verde la lesión derecha y en rosa la lesión izquierda, en turquesa el ventrículo izquierdo y en azul rey, el ventrículo derecho.

a valores elevados de interleucina 8 , una citocina proinflamatoria, en suero materno durante el segundo y el tercer trimestres, y el aumento del volumen de líquido cefalorraquídeo ventricular, así como la disminución del volumen cortical entorrinal izquierdo, característicos de la esquizofrenia ${ }^{12}$. Estos estudios apoyan la hipótesis de que la microglía es inducida por inflamación prenatal involucrada en la fisiopatología de la esquizofrenia ${ }^{15}$.

A pesar de que la lesión en el hipocampo ventral se realizó de forma bilateral, observamos cualitativamente un mayor tamaño de la lesión del hemisferio izquierdo en la mayoría de las mediciones. Posiblemente el cambio en el tamaño de la lesión sea un mecanismo de compensación en el grupo de LNHV, retrasando la destrucción simétrica de regiones circundantes al hipocampo ventral y restituyendo el tejido circundante a la lesión en edades mayores, posteriores al inicio sintomático de la esquizofrenia ${ }^{16-18}$. Además de la asimetría observada en la lesión, encontramos que posiblemente el pico máximo en el tamaño de esta ocurre en el día PD96, y a partir de ahí el tamaño de la lesión se revierte. Este hallazgo puede sugerir la presencia de una neurogénesis aberrante, como ya comentamos en otro estudio ${ }^{19}$, cuando se caracterizó la conducta tipo esquizofrenia y se realizaron cortes de cerebro; sin embargo, para respaldar esta idea habría que realizar otro tipo de pruebas. Se ha hipotetizado que el mecanismo de este aumento de la densidad neuronal implica el atrapamiento de neuronas que migran de la matriz germinal a la corteza, debido a la lesión ${ }^{20,21}$. Las diferencias cualitativas en los volúmenes ventriculares, así como en el hipocampo, fueron identificadas a partir del PD69 en el grupo con LNHV, en comparación con el grupo control. En el modelo animal, este día marca el término de la adolescencia y el inicio de la edad adulta, lo cual es consistente con la aparición de los síntomas de esquizofrenia predominantemente en esta etapa en el ser humano ${ }^{8}$. Sumado a la posible homología temporal de aparición de los síntomas entre el modelo y los humanos, también encontramos diferencias cualitativas en el hipocampo que podrían estar involucradas en el desarrollo de la sintomatología cognitiva y las alteraciones de la memoria que se pueden observar en algunos pacientes con esquizofrenia ${ }^{22,23}$. En este sentido, el modelo de LNHV podría utilizarse para probar nuevas aproximaciones terapéuticas, como la estimulación cerebral profunda, para el tratamiento futuro de los pacientes con esquizofrenia ${ }^{24-26}$.

Los resultados destacan la importancia del uso del modelo animal como un enfoque viable y valioso para 
el estudio de las enfermedades psiquiátricas. Los rasgos de la esquizofrenia que se han reproducido con éxito con el modelo LNHV proporcionan información sobre los mecanismos neuronales que podrían estar involucrados en la sintomatología de la enfermedad. Además, podrían ser fuente de futuras investigaciones traslacionales y posibles marcadores imagenológicos de psicosis. Como ejemplo podríamos citar la crisis del lóbulo temporal medial, por la que muchos pacientes presentan manifestaciones de psicosis (síntomas característicos de la esquizofrenia y que son emulados por el modelo de LNHV) ${ }^{27,28}$. Consideramos importante evaluar si los pacientes afectados por crisis del lóbulo temporal medial y psicosis podrían presentar alteraciones cerebrales homologas a las del modelo, en el sentido de tener un marcador de psicosis en este grupo particular de pacientes.

\section{Limitantes y perspectivas}

Es importante mencionar que, aunque la RM es la mejor técnica para estudiar estructuralmente el cerebro in vivo, durante la realización de este estudio tuvimos algunas dificultades que podrían ser consideradas por otros grupos de trabajo al realizar estudios similares; por ejemplo, la muerte de algunos roedores durante o después de nuestros experimentos, probablemente debido a su corta edad, o la hipotermia inducida por el equipo de medición, no tomada en cuenta, hasta que los animales fueron expuestos durante la adquisición de las imágenes. En referencia a este punto, solo pudimos continuar el seguimiento de dos animales durante todo el estudio, por lo que se volvió imposible realizar estimaciones estadísticas, transformando nuestro trabajo en un estudio cualitativo y exploratorio. Otra limitante, la cual podría impactar directamente en la asimetría observada de la lesión, podría deberse a un error sistemático, por lo que recomendamos la aleatorización de los hemisferios durante la cirugía, además de poder aleatorizar dos personas que realicen la cirugía para minimizar el posible error.

\section{Conclusiones}

Observamos una posible homología en el agrandamiento cualitativo de los ventrículos en el modelo animal de LNHV, como lo reportado previamente en pacientes con esquizofrenia, además de la presencia de una posible gliosis alrededor de la lesión en el modelo de LNHV.

\section{Agradecimientos}

Nuestro agradecimiento al Centro Nacional de Imagenología e Instrumentación Médica (CI3M), de la Universidad Autónoma Metropolitana, por las facilidades prestadas.

\section{Financiamiento}

Instituto Nacional de Medicina Genómica 12/2014/I

\section{Conflicto de intereses}

Los autores declaran no tener conflictos de intereses.

\section{Responsabilidades éticas}

Protección de personas y animales. Los autores declaran que los procedimientos seguidos se conformaron a las normas éticas del comité de experimentación humana responsable y de acuerdo con la Asociación Médica Mundial y la Declaración de Helsinki.

Confidencialidad de los datos. Los autores declaran que han seguido los protocolos de su centro de trabajo sobre la publicación de datos de pacientes.

Derecho a la privacidad y consentimiento informado. Los autores han obtenido el consentimiento informado de los pacientes y/o sujetos referidos en el artículo. Este documento obra en poder del autor de correspondencia.

\section{Bibliografía}

1. Whiteford HA, Ferrari AJ, Degenhardt L, Feigin V, Vos T. The global burden of mental, neurological and substance use disorders: an analysis from the Global Burden of Disease Study 2010. PLoS One. 2015;10:e0116820.

2. Medina-Mora ME, Borges G, Benjet C, Lara C, Berglund P. Psychiatric disorders in Mexico: lifetime prevalence in a nationally representative sample. Br J Psychiatry. 2007;190:521-8.

3. Cancel A, Dallel S, Zine A, El-Hage W, Fakra E. Understanding the link between childhood trauma and schizophrenia: a systematic review of neuroimaging studies. Neurosci Biobehav Rev. 2019;107:492-504.

4. Andreasen NC, Nopoulos P, Magnotta V, Pierson R, Ziebell S, Ho BC. Progressive brain change in schizophrenia: a prospective longitudinal study of first-episode schizophrenia. Biol Psychiatry. 2011;70:672-9.

5. Sams-Dodd F, Lipska BK, Weinberger DR. Neonatal lesions of the rat ventral hippocampus result in hyperlocomotion and deficits in social behaviour in adulthood. Psychopharmacology (Berl). 1997;132:303-10.

6. Genis-Mendoza A, López-Rubalcava C. ¿Es posible modelar esquizofrenia en un modelo animal? El Residente. 2011;6:120-6. Disponible en: https://www.medigraphic.com/pdfs/residente/rr-2011/rr112i.pdf

7. Genis-Mendoza AD, Beltrán-Villalobos I, Nicolini H. Evaluación conductual del fenotipo «esquizofrenia» en un modelo animal por lesión neonatal del hipocampo ventral en ratas juveniles y adultas. Gac Med Mex. 2014;150:420-31.

8. Genis-Mendoza AD, Beltrán-Villalobos I, Nicolini-Sánchez H. [Behavioral assessment of the "schizophrenia-like" phenotype in an animal model of neonatal lesion in the ventral hippocampus (NLVH) of young and adult rats]. Gac Med Mex. 2014;150:420-31. 
9. Bouilleret V, Nehlig A, Marescaux C, Namer IJ. Magnetic resonance imaging follow-up of progressive hippocampal changes in a mouse model of mesial temporal lobe epilepsy. Epilepsia. 2000;41:642-50.

10. Bertrand JB, Langlois JB, Bégou M, Volle J, Brun P, D'Amato T, et al. Longitudinal MRI monitoring of brain damage in the neonatal ventral hippocampal lesion rat model of schizophrenia. Hippocampus. 2010; 20:264-78.

11. Najjar S, Pearlman DM. Neuroinflammation and white matter pathology in schizophrenia: systematic review. Schizophrenia Research. 2015;61:102-12.

12. Feigenson KA, Kusnecov AW, Silverstein SM. Inflammation and the two-hit hypothesis of schizophrenia. Neurosci Biobehav Rev. 2014; 38:72-93.

13. Müller N, Weidinger $E$, Leitner B, Schwarz MJ. The role of inflammation in schizophrenia. Frontiers in Neuroscience. 2015:9:372.

14. Müller N. Inflammation in schizophrenia: pathogenetic aspects and therapeutic considerations. Schizophr Bull. 2018;44:973-82.

15. McFarquhar M, Elliott R, McKie S, Thomas E, Downey D, Mekli K, et al. TOMM40 rs2075650 may represent a new candidate gene for vulnerability to major depressive disorder. Neuropsychopharmacology. 2014; 39:1743-53.

16. Sekar A, Bialas AR, De Rivera H, Davis A, Hammond TR, Kamitaki N, et al. Schizophrenia risk from complex variation of complement component 4. Nature. 2016;530:177-83.

17. Lipska BK, Weinberger DR. Delayed effects of neonatal hippocampal damage on haloperidol-induced catalepsy and apomorphine-induced stereotypic behaviors in the rat. Dev Brain Res. 1993;75:213-22.

18. De Bellis MD, Keshavan MS, Beers SR, Hall J, Frustaci K, Masalehdan A et al. Sex differences in brain maturation during childhood and adolescence. Cereb Cortex. 2001;11:552-7.
19. Genis-Mendoza AD, Gallegos-Silva RI, López-Casamichana M, López-Rubalcava C, Nicolini H. Gene expression profiles of nucleus accumbens, prefrontal cortex and hippocampus in an animal model of schizophrenia: proposed candidate genes. Actas Esp Psiquiatr. 2014;41:154-63.

20. Bandeira ID, Barouh JL, Bandeira ID, Quarantini L. Analysis of the superior temporal gyrus as a possible biomarker in schizophrenia using voxel-based morphometry of the brain magnetic resonance imaging: a comprehensive review. CNS Spectr. 2021;26:319-25.

21. Meade T, Sleight $P$, Collins R, Armitage J, Parish S, Barton J, et al. SLCO1B1 variants and statin-induced myopathy - a genomewide study. N Engl J Med. 2008;359:789-99.

22. Heckers S, Konradi C. Hippocampal neurons in schizophrenia. J Neural Transm. 2002;109:891-905.

23. Hall J, Whalley HC, Marwick K, McKirdy J, Sussmann J, Romaniuk L, et al. Hippocampal function in schizophrenia and bipolar disorder. Psychol Med. 2010;40:761-70.

24. Agarwal P, Sarris CE, Herschman Y, Agarwal N, Mammis A. Schizophrenia and neurosurgery: a dark past with hope of a brighter future. J Clin Neurosci. 2016;34:53-8.

25. Robison RA, Taghva A, Liu CY, Apuzzo MLJ. Surgery of the mind, mood, and conscious state: an idea in evolution. World Neurosurg. 2013;80:S2-26.

26. Luigjes J, De Kwaasteniet BP, De Koning PP, Oudijn MS, Van Den Munckhof $\mathrm{P}$, Schuurman PR, et al. Surgery for psychiatric disorders. World Neurosurg. 2013;80:S31.e17-28.

27. Flornhenry P. Psychosis and temporal lobe epilepsy; a controlled investigation. Epilepsia. 1969;10:363-95.

28. Kandratavicius L, Lopes-Aguiar C, Bueno-Júnior LS, Romcy-Pereira RN, Hallak JEC, Leite JP. Comorbidades psiquiátricas na epilepsia do lobo temporal: possíveis relações entre desordens psicóticas e comprometimento de circuitos límbicos. Rev Bras Psiquiatr. 2012;34:454-66. 